\title{
Synthesis of New Single CFOA-Based VCOs Incorporating the Voltage Summing Property of Analog Multipliers
}

\author{
S. S. Gupta, ${ }^{1}$ D. R. Bhaskar, ${ }^{2}$ and R. Senani ${ }^{3}$ \\ ${ }^{1}$ Department of Industrial Policy and Promotion, Ministry of Commerce and Industry, Government of India, Udyog Bhawan, \\ New Delhi 110011, India \\ ${ }^{2}$ Department of Electronics and Communication Engineering, Faculty of Engineering and Technology, Jamia Millia Islamia, \\ New Delhi 110025, India \\ ${ }^{3}$ Division of Electronics and Communication Engineering, Netaji Subhas Institute of Technology, Sector 3, Dwarka, \\ New Delhi 110078, India
}

Correspondence should be addressed to R. Senani, senani@nsit.ac.in

Received 26 May 2012; Accepted 4 July 2012

Academic Editors: S. Gift, D. Rossi, and E. Tlelo-Cuautle

Copyright ( 12012 S. S. Gupta et al. This is an open access article distributed under the Creative Commons Attribution License, which permits unrestricted use, distribution, and reproduction in any medium, provided the original work is properly cited.

\begin{abstract}
Recently, current feedback operational amplifier (CFOA) and analog multiplier-(AM-) based-voltage controlled oscillators (VCOs) have been published in the literature which require 2 CFOAs and 2 AMs for linear tuning law between control voltage and frequency of oscillation. In this paper, a family of eight new voltage-controlled oscillators (VCOs), with linear tuning laws, employing only a single CFOA in conjunction with two analog multipliers (AMs), has been derived through a systematic state-variable methodology. This has been made possible by exploiting the voltage summing property of the multiplier chosen which has never been done in the literature earlier. The workability of the presented VCOs has been verified by experimental results based on AD844 type CFOAs and AD534 type AMs, and the advantages of new circuits over the previously known CFOA-AM-based VCOs have been highlighted.
\end{abstract}

\section{Introduction}

Although a number of new building blocks and concepts related to current-mode circuits have been investigated in the literature [1-3], the use of current feedback operational amplifiers (CFOAs) as an alternative to the traditional voltage-mode op-amps (VOAs) has attracted considerable attention (see [4-7] and the references cited therein) in various instrumentation, signal processing, and signal generation applications due to their commercial availability as off-the-shelf ICs as well as due to the well-known advantages offered by CFOAs over the VOAs [4-6]. Because of these reasons, the use of CFOAs has been extensively investigated in realizing oscillators, for instance, see $[6,8-11]$ and the references cited therein. Although a variety of CFOAs are available from various manufacturers, AD844 (from Analog Devices), which contains a CCII+ followed by a voltage buffer is particularly flexible and popular due to the availability of $z$-terminal of the CCII+ therein as an externally accessible lead which permits AD844 to be used as a CCII+ (one AD844) or as CCII-(realizable with two AD844s) or as a general 4-terminal building block [6].

This paper is concerned with the use of CFOAs in the realization of voltage-controlled oscillators (VCOs) which are required in several instrumentation, electronic, and communication systems, such as in function generators, in production of electronic music to generate variable tones, in phase locked loops, and in frequency synthesizers [12-17].

Although, recently, the use of CFOAs in the realization of linear VCOs has been reported in [7, 11], in this paper, the voltage summing property of the commercially available analog multiplier (AM) AD 534 has been exploited to yield new VCO circuits possessing properties not available in the previously known CFOA-AM-based VCOs. It may be recalled that, in [7], all existing two CFOA-based single resistance controlled oscillators (SRCOs) have been shown to be convertible into VCOs by using FET-based linearized voltage-controlled resistor (VCR), thereby, leading to VCO 
circuits employing two to three CFOAs, however, in such circuits, the tuning law between oscillation frequency and control voltage is not linear.

More recently, a number of CFOA-based VCOs have been proposed in the literature $[11,18]$, in which the VCO realization has been achieved by devising a CFOAbased RC-active oscillator with two analog multipliers (AMs) appropriately embedded to enable independent control of the oscillation frequency through an external control voltage $V_{C}$ applied as a common multiplicative input to both the multipliers such that oscillation frequency becomes a linear function of $V_{C}$, that is,

$$
f_{0} \propto V_{C}
$$

whereas VCO circuits of [11] all employ only two CFOAs, by contrast, two VCOs reported in [18] employ only a single CFOA along with three resistors, two grounded capacitors, and two AMs. These circuits, however, suffer from the difficulty of employing a variable capacitance for adjusting the circuits to produce oscillations, which is not a very desirable option. Another disadvantage of the circuits of [18] is the requirement of matching of two resistor values which is also not desirable since any mismatch in component values may change the frequency of oscillation or disturb the oscillations altogether.

The object of this paper is to present a family of eight new single CFOA-based VCOs which remove the above mentioned difficulties while employing a bare minimum number of active and passive components, namely, only one CFOA, only two multipliers (essential for obtaining linear control of oscillation frequency), two/three resistors, and two capacitors. Experimental results using AD844 type CFOAs and AD534 type AMs have been given which confirm the practical viability of the proposed circuits. The advantages of the new CFOA-based VCOs as compared to the previously known CFOA-based VCOs are highlighted.

\section{Proposed VCO Configurations}

AD844 type CFOA is a 4-port analog building block with $x, y$ being the input terminals and $z$ and $w$ being output terminals, which is characterized by the terminal equations $i_{y}=0, v_{x}=v_{y}, i_{z}=i_{x}, v_{w}=v_{z}$ and its symbolic notation is shown in Figure 1(a). The AD534 type AM is a 4-port building block with three differential inputs (shown as $V_{1}$, $V_{2}$, and $V_{Z 1}$ in the present case) and one output $V_{0}$ and is characterized by $V_{0}=K\left(V_{1} V_{2} / V_{\text {ref }}\right)+V_{Z}$ (symbolic notation in Figure 1(b)), where $V_{1}$ and $V_{2}$ are two inputs, $V_{\text {ref }}$ is the reference voltage set internally, usually at 10 volts in case of AD534 and $K$ can be set up +1 or -1 by grounding appropriate input terminals, and $V_{Z}$ is the voltage applied at the input terminal of AM which appears at the output without any multiplying factor.

Since the new VCO circuits derived in the present paper have been synthesized using the state-variable methodology introduced earlier in $[8,9]$, we first briefly outline the relevant basic ideas.

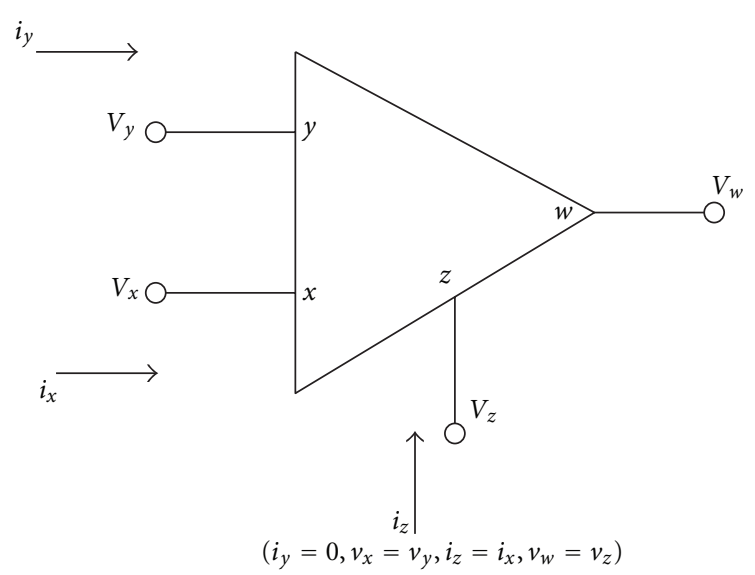

(a)

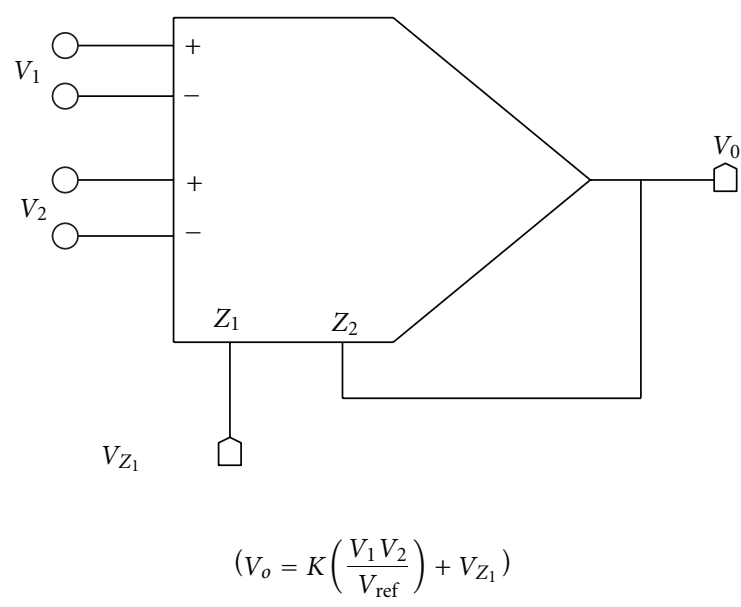

(b)

FIGURE 1: Symbolic notations: (a) CFOA and (b) analog multiplier.

A canonic second-order (i.e., employing only two capacitors) oscillator can, in general, be characterized by the following autonomous state equation:

$$
\left[\begin{array}{l}
\dot{x}_{1} \\
\dot{x_{2}}
\end{array}\right]=\left[\begin{array}{ll}
a_{11} & a_{12} \\
a_{21} & a_{22}
\end{array}\right]\left[\begin{array}{l}
x_{1} \\
x_{2}
\end{array}\right]
$$

where $x_{1}$ and $x_{2}$ are the state variables representing the voltage across the two capacitors employed in the oscillator.

From the above, the characteristic equation (CE) $s^{2}-$ $\left(a_{11}+a_{22}\right) s+\left(a_{11} a_{22}-a_{12} a_{21}\right)=0$ gives the condition of oscillation (CO) and frequency of oscillation (FO) as follows:

$$
\begin{gathered}
\text { CO: }\left(a_{11}+a_{22}\right)=0, \\
\text { FO: } \omega_{0}=\sqrt{\left(a_{11} a_{22}-a_{12} a_{21}\right)} .
\end{gathered}
$$

The state-variable methodology consists of the following steps:

(i) a selection of the parameters $a_{i j}, i=1,2 ; j=1,2$, in accordance with the required features (e.g., noninteracting controls for frequency of oscillation and condition of oscillation through separate resistors), 


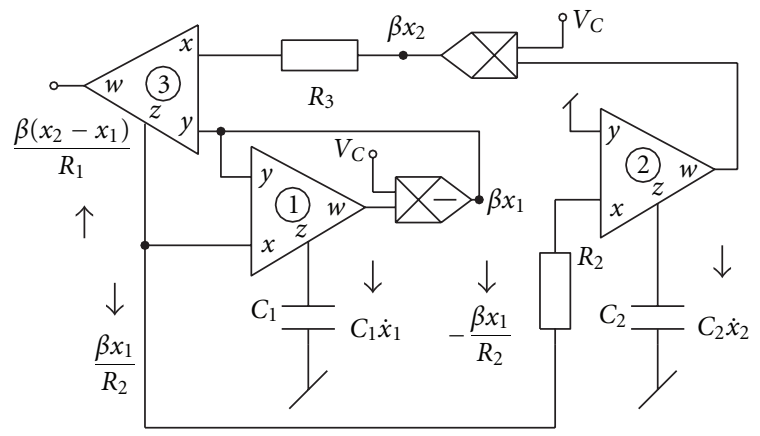

FIgURE 2: VCO derived from matrix $[A]_{2}\left(\mathrm{CO}: R_{1}=R_{2}\right.$, FO: $f_{0}=$ $\left.(\beta / 2 \pi) \sqrt{1 / C_{1} C_{2} R_{1} R_{2}}\right)$.

(ii) conversion of the resulting state equations into node equations (NE), and, finally,

(iii) constitution of a physical circuit from these node equations.

Different circuits are expected to be generated by making different choices of the parameters $a_{11}, a_{12}, a_{21}$, and $a_{22}$. For noninteractive controls of condition of oscillation and frequency of oscillation, let us assume that $\mathrm{CO}$ is to be controlled by a resistor $R_{1}$ (independent of some resistor $R_{2}$ ) and that FO is to be controlled by the resistor $R_{2}$ (independent of the resistor $R_{1}$; with a third resistor $R_{3}$ featuring in both $\mathrm{CO}$ as well as in FO). These conditions lead to the following requirements:

(a) The expression of $\left(a_{11}+a_{22}\right)$ should either not have terms containing $R_{2}$ or they should be cancelled out. Thus, in $\left(a_{11}+a_{22}\right)$, there should be two terms left with opposite signs involving $R_{1}$ and $R_{3}$.

(b) Similarly, to have FO independent of $R_{1}$, the expression $\left(a_{11} a_{22}-a_{12} a_{21}\right)$ should either not have the terms containing $R_{1}$ or they should be cancelled out. Thus, FO should be a function of resistors $R_{2}$ and $R_{3}$ only (along with $C_{1}$ and $C_{2}$ ).

Based upon the above considerations, let us construct the required $[A]$ matrix in the following form:

$$
[A]_{1}=\left[\begin{array}{cc}
\frac{1}{C_{1}}\left(\frac{1}{R_{3}}-\frac{1}{R_{1}}\right) & \frac{1}{C_{1} R_{2}} \\
-\frac{1}{C_{2} R_{3}} & 0
\end{array}\right] .
$$

It is readily seen that the above matrix fulfils both the requirements $(a)$ and $(b)$ above.

The CE formulated from the above matrix gives the following $\mathrm{CO}$ and $\mathrm{FO}$ :

$$
\begin{gathered}
\text { CO: } R_{1}=R_{3}, \\
\text { FO: } \omega_{0}=\frac{1}{\sqrt{C_{1} C_{2} R_{2} R_{3}}} .
\end{gathered}
$$

Since the oscillators to be derived have to incorporate at least two analog multipliers in order to provide linear control of oscillation frequency through an external control voltage $V_{C}$ which is applied in place of the second input $V_{2}$ of the multipliers (to be applied as a common multiplicative input to both the analog multipliers), step (i) - selection of the matrix parameters - outlined above needs to be modified to include the term $\beta\left(\beta=V_{C} / V_{\text {ref }}\right)$. It should be done in such a way that the final expression of the CO does not contain the term $\beta$, but the expression of FO is modified to $f_{0}=\beta / 2 \pi \sqrt{C_{1} C_{2} R_{2} R_{3}}$ so that we can get $f_{0} \propto \beta$, and hence, $f_{0} \propto V_{C}$.

Parameters of the matrix $[A]_{1}$ given in (4) can now be modified in one of the following ways:

(i) by including $\beta^{2}$ as a factor of $a_{12}$ or $a_{21}$,

(ii) $\beta$ as a factor $a_{12}$ as well as that of $a_{21}$,

(iii) $\beta$ as a factor in all the parameters of matrix $[A]$.

When we do so, we can manage independent control of FO by $\beta$ and that of CO by $R_{1}$ or $R_{2}$ and we need to employ only two resistors.

Using the modification (iii) and employing only $R_{1}$ and $R_{2}$, matrix $[A]_{1}$ of $(4)$ can be written as follows:

$$
[A]_{2}=\left[\begin{array}{cc}
\frac{\beta}{C_{1}}\left(\frac{1}{R_{2}}-\frac{1}{R_{1}}\right) & \frac{\beta}{C_{1} R_{1}} \\
-\frac{\beta}{C_{2} R_{2}} & 0
\end{array}\right] .
$$

From matrix $[A]_{2}$, we get the following node equations:

$$
\begin{gathered}
C_{1} \dot{x}_{1}=\frac{\beta\left(x_{2}-x_{1}\right)}{R_{1}}+\frac{\beta x_{1}}{R_{2}}, \\
C_{2} \dot{x}_{2}=-\frac{\beta x_{1}}{R_{2}} .
\end{gathered}
$$

Implementation of the above NEs employing two multipliers results in the circuit shown in Figure 2. Various current components of (7) and (8) have been marked in the circuit to make the synthesis clear.

It may be noted that here we require three CFOAs along with two AMs to implement NEs of (7) and (8). We now show that by an alternative representation of (7) and (8) and an appropriate incorporation of the $z$-terminal of AM, it becomes possible to implement the modified NEs with only a single CFOA. Let us add (7) and (8) to create a new equation $((9)$ in the following) while we keep (8) as it is (shown as (10) in the following):

$$
\begin{aligned}
C_{1} \dot{x}_{1}+C_{2} \dot{x}_{2} & =\frac{\beta\left(x_{2}-x_{1}\right)}{R_{1}}, \\
C_{2} \dot{x}_{2} & =-\frac{\beta x_{1}}{R_{2}} .
\end{aligned}
$$

It is interesting to note that when the voltage summing property of the AMs is effectively utilized, the implementation of (9) and (10) then leads to a different circuit (shown as VCO1 in Figure 3 ) which requires only a single CFOA in contrast to the circuit of Figure 2 needing three CFOAs. 
It may be noted that the value of $K$ for both AM1 and AM2 has been chosen as -1 . An alternative circuit can be generated if we take $K=+1$ for AM1 and $K=-1$. It will facilitate the grounding of the resistor $R_{2}$ and the result will be VCO-2 in Figure 3. If we try to connect the grounded capacitor $C_{1}$ at the $z$-terminal of CFOA and taking $K=1$ for AM1 and AM2, VCO-3 of Figure 3 can be generated. In an effort to ground $R_{1}$ by taking $K=-1$ for AM1 and $K=1$ for AM1, we obtain VCO-4, Figure 3.

We have found that in addition to (6), the following matrices are suitable for the synthesis of such kind of VCOs:

$$
\begin{gathered}
{[A]_{3}=\left[\begin{array}{cc}
\frac{\beta}{C_{1}}\left(\frac{1}{R_{2}}-\frac{2}{R_{1}}\right) & \frac{\beta}{C_{1} R_{1}} \\
-\frac{\beta}{C_{2} R_{2}} & 0
\end{array}\right],} \\
{[A]_{4}=\left[\begin{array}{cc}
\frac{\beta}{C_{1} R_{1}} & -\frac{\beta}{C_{1} R_{1}} \\
\frac{2 \beta}{C_{2} R_{2}} & -\frac{\beta}{C_{2} R_{2}}
\end{array}\right],} \\
{[A]_{5}=\left[\begin{array}{cc}
\frac{\beta^{2}}{C_{1} R_{1}} & -\frac{\beta^{2}}{C_{1} R_{1}} \\
\frac{1+\beta^{2}}{C_{2} R_{2}} & -\frac{\beta^{2}}{C_{2} R_{2}}
\end{array}\right],} \\
{[A]_{6}=\left[\begin{array}{cc}
0 & \frac{\beta}{C_{1} R_{2}} \\
-\frac{\beta}{C_{2} R_{3}} & \frac{1}{C_{2}}\left(\frac{1}{R_{3}}-\frac{1}{R_{1}}\right)
\end{array}\right] .}
\end{gathered}
$$

Based on the state variable methodology explained above, along with any or all the modifications (i)-(iii) suggested above, a number of VCOs can be generated from the matrices $[A]_{3}-[A]_{6}$ given in (11), and the resulting circuits along with their $\mathrm{CO}$ and FO are given in Figures 4, 5,6 , and 7 .

It may be emphasized that in the generated VCOs of Figure 3 to Figure 6, it has been possible to reduce the number of CFOAs to the bare minimum, that is, only one by exploiting the feature of summation of a third input $Z$ in the output of the AD534 type multipliers which has been appropriately incorporated in the node equations to synthesize the VCOs. Furthermore, it should also be observed that none of single CFOA-based VCOs derived here are obtainable from the single CFOA-based SRCOs [19-26]. This can be understood by noting that the earlier single-CFOA SRCOs typically require three resistors and two capacitors, whereas the new VCOs 1-7 employ only two resistors and two capacitors. On the other hand, whereas VCOs 6-8 employ both grounded capacitors, not even a single SRCO is known so far [19-26] which has both grounded capacitors. In view of the above, it is clear that the circuits derived here are new and unique of their type, which has never been presented in the literature earlier.

It now appears worthwhile to compare various features of the eight VCOs synthesized in this section. In this context, the following may be noted. (i) Out of the eight circuits presented here, VCOs 1-7 employed two Rs and two Cs out of which VCOs 1-5 do not employ both grounded capacitors. Nevertheless, they offer complete independence of $f_{0}$ controlled by $\beta$ after $\mathrm{CO}$ having been adjusted by $R_{1}$ and/or $R_{2}$. In this context, VCOs 2, 4 have one of the $\mathrm{CO}$ controlling resistors grounded which is considered advantageous from the viewpoint of incorporating amplitude stabilization/control circuitry.

(ii) VCOs 6 and 7 offer the use of both grounded capacitors, and, out of these, VCO-7 also has one of the COs controlling resistor $R_{2}$ grounded.

(iii) The VCO- 8 may be considered to be the only one which possesses simultaneously almost all the desirable features, namely, completely noninteracting control of CO through $R_{1}$, the CO controlling resistor being grounded, the employment of both grounded capacitors, and an additional degree of freedom via $R_{2}$ to scale up or down the frequency $f_{0}$ which is otherwise linearly controllable by $\beta$.

From (i)-(iii) above, it is seen that from the point of view of the various features considered above, the VCO- 8 is the best circuit of the entire set.

\section{Consideration of CFOA Parasitics}

The prominent nonidealities of the CFOAs include- - a finite nonzero input resistance $R_{x}$ at port- $x$ (typically around $50 \Omega$ ), $y$-port parasitics consisting of a parasitic resistance $R_{y}$ (typically $2 \mathrm{M} \Omega$ ) in parallel with a parasitic capacitance $C_{y}$ (typically $2 \mathrm{pF}$ ), and $z$-port parasitic impedance consisting of a parasitic resistance $R_{p}$ (typically $3 \mathrm{M} \Omega$ ) in parallel with a parasitic capacitance $C_{p}$ (typically between $4-5 \mathrm{pF}$ ). In case of an analog multiplier, the finite non-zero output resistance $r_{\text {out }}$, as per datasheet of AD534, is merely $1 \Omega$ and hence can be ignored in all the cases. On the other hand, the input impedance of the AM, being $10 \mathrm{M} \Omega$, is sufficiently high and, hence, its effect can be ignored.

For a quantitative assessment of the effect of the various CFOA non-idealities, we have carried out an analysis of all the VCOs with parasitics included, and the nonideal expressions for $\mathrm{CO}$ and $\mathrm{FO}$ are given in Tables 1 and 2, respectively. It may be noted that in all the VCOs, the $y$-port parasitics of CFOA do not affect the operation of the circuits either due to y-terminal being grounded or connected to output terminal of AM and hence do not appear in the nonideal expressions of $\mathrm{CO}$ and $\mathrm{FO}$.

From the expressions of the oscillation frequencies for the various VCOs, it is easy to visualize that all the sensitivity coefficients would be $-1 / 2$, and the circuits thus enjoy very low sensitivity to component tolerances.

It is easy to infer from the expressions given in Tables 1 and 2 that the errors caused by the influence of CFOA can be kept small by choosing all external resistors to be much larger than $R_{x}$ but much smaller than $R_{p}$ and choosing both external capacitors to be much larger than $C_{p}$.

It is observed from Tables 1 and 2 that in case of VCO-8, CO controlling element $R_{1}$ does not appear in the nonideal 


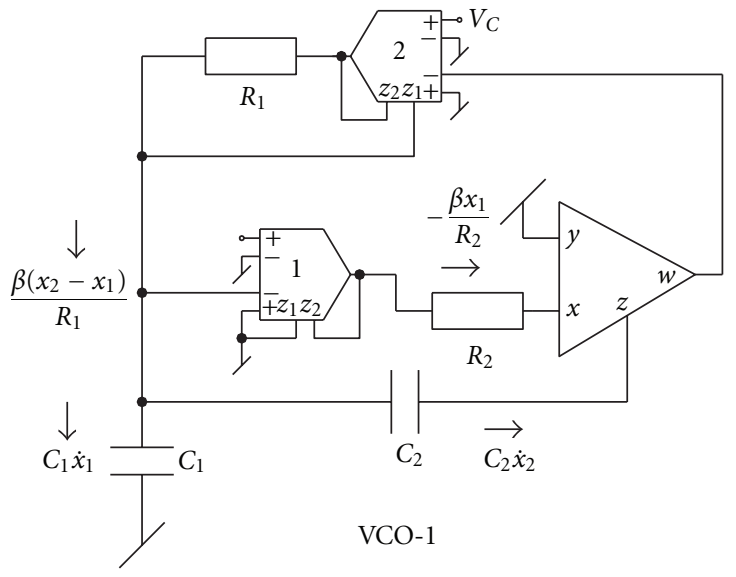

(a)

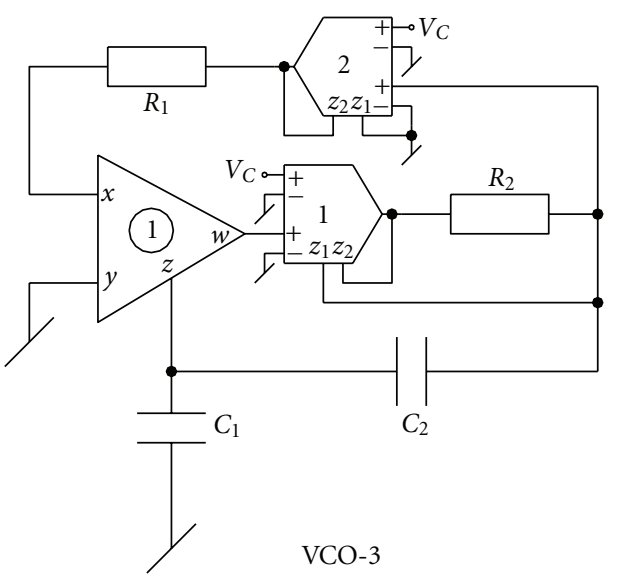

(c)

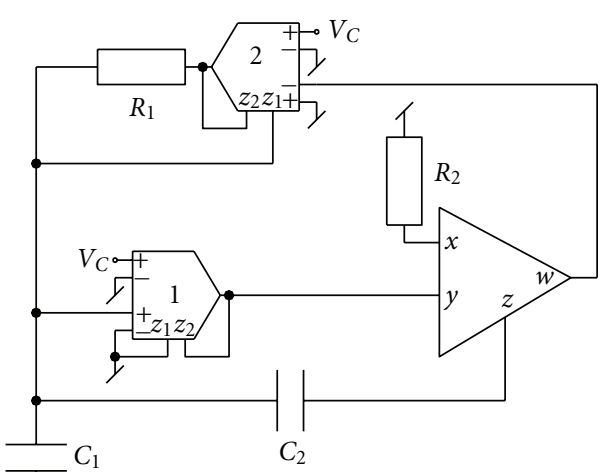

VCO-2

(b)

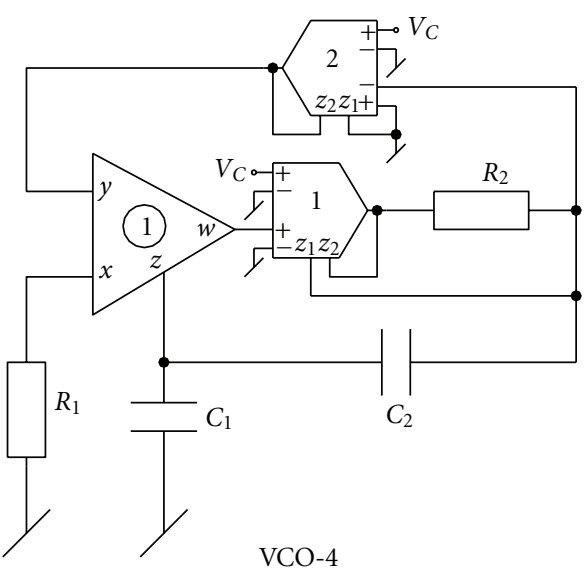

(d)

FIGURe 3: VCOs derived from matrix $[A]_{2}\left(\mathrm{CO}: R_{1}=R_{2}\right.$, FO: $\left.f_{0}=(\beta / 2 \pi) \sqrt{1 / C_{1} C_{2} R_{1} R_{2}}\right)$.

TABLE 1: Ideal and nonideal conditions of oscillation for the new VCOs.

\begin{tabular}{lcc}
\hline VCO & Ideal CO & Nonideal CO \\
\hline 1,2 & $R_{1}=R_{2}$ & $R_{1}=R_{2} \frac{\left(1+R_{x} / R_{2}\right)}{\left\{1-\left(1 / \beta C_{2} R_{p}\right)\left(R_{2}+R_{x}\right)\left(C_{1}+C_{2}\right)\right\}}$ \\
3,4 & $R_{1}=R_{2}$ & $R_{1}=R_{2}\left\{\frac{1}{\left(1-R_{2} / \beta R_{p}\right)}-\frac{R_{x}}{R_{2}}\right\}$ \\
5 & $R_{1}=2 R_{2}$ & $R_{1}=2 R_{2}\left\{\frac{1}{\left(1-R_{2} / \beta R_{p}\right)}-\frac{R_{x}}{2 R_{2}}\right\}$ \\
6 & $R_{1}=\frac{C_{2}}{C_{1}} R_{2}$ & $R_{1}=\frac{C_{2}}{C_{1}} R_{2}\left\{\frac{1+R_{x} / R_{2}}{1+\left(1 / \beta R_{p}\right)\left(R_{2}+R_{x}\right)}\right\}$ \\
7 & $R_{1}=\frac{C_{2}}{C_{1}} R_{2}$ & $R_{1}=\frac{C_{2}}{C_{1}} R_{2}\left\{\frac{\left(1+C_{p} / C_{2}\right)\left(1+R_{x} / R_{2}\right)}{1+\left(1 / \beta^{2} R_{p}\right)\left(R_{2}+R_{x}\right)}\right\}$ \\
8 & $R_{1}=R_{3}$ & $R_{1}=R_{3}\left\{\frac{1+R_{x} / R_{3}}{1-\left(1 / R_{p}\right)\left(R_{3}+R_{x}\right)}\right\}$
\end{tabular}

expression of FO, and FO controlling elements $R_{2}$ and $\beta$ do not appear in the nonideal expression of $\mathrm{CO}$. This means that independent control of $\mathrm{CO}$ and $\mathrm{FO}$ remains intact for VCO8 even after consideration of the parasitics. Hence, VCO- 8 is the best circuit of the lot from this viewpoint.

\section{Experimental Results}

All the VCOs have been experimentally studied using AD844 type CFOAs and AD534 type AMs biased with \pm 12 volts DC power supplies. In the experimental studies, the component values chosen were as follows: for VCOs 1-4, 6, $7 R_{1}=R_{2}=$ $2 \mathrm{k} \Omega$, those for VCO-5 were chosen as $R_{1}=2 \mathrm{k} \Omega, R_{2}=$ $1 \mathrm{k} \Omega$, and for VCO- $8 R_{1}=R_{2}=R_{3}=1 \mathrm{k} \Omega$. Capacitor values for all the VCOs were taken as $C_{1}=C_{2}=1 \mathrm{nF}$. All the circuits have been found to work as predicted by theory, and it has been possible to generate oscillation frequencies from tens of $\mathrm{kHz}$ to several hundreds of $\mathrm{kHz}$ with tolerable errors in the frequency (whose values can be computed using the expressions given in the last column of Table 2). The maximum frequencies realizable with the VCOs $1-7$ have been of the order of $72.85 \mathrm{kHz}$, whereas in case of VCO- 8 the maximum attainable frequency was $137.5 \mathrm{kHz}$.

In the absence of an automatic amplitude control, it is normally expected that the amplitude of oscillation would 
TABLE 2: Ideal and nonideal frequency of oscillation.

\begin{tabular}{|c|c|c|c|c|}
\hline $\mathrm{VCO}$ & Ideal FO & \multicolumn{3}{|c|}{ Nonideal FO } \\
\hline 1,2 & $f_{0}=\frac{\beta}{2 \pi} \sqrt{\frac{1}{C_{1} C_{2} R_{1} R_{2}}}$ & $f_{0}=\frac{\beta}{2 \pi} \sqrt{C_{1} C}$ & $\frac{1}{{ }_{2} R_{1} R_{2}}\left\{\frac{}{(1}\right.$ & $\frac{1}{\left.+C_{p} / C_{1}+C_{p} / C_{2}\right)\left(1+R_{x} / R_{2}\right)}$ \\
\hline $3,4,5$ & $f_{0}=\frac{\beta}{2 \pi} \sqrt{\frac{1}{C_{1} C_{2} R_{1} R_{2}}}$ & $f_{0}=\frac{\beta}{2 \pi}$ & $\frac{1}{C_{1} C_{2} R_{1} R_{2}}$ & $\left.\frac{1}{\left(1+C_{p} / C_{1}\right)\left(1+R_{x} / R_{1}\right)}\right\}$ \\
\hline 6 & $f_{0}=\frac{\beta}{2 \pi} \sqrt{\frac{1}{C_{1} C_{2} R_{1} R_{2}}}$ & $f_{0}=\frac{\beta}{2 \pi}$ & $\frac{1}{C_{1} C_{2} R_{1} R_{2}}$ & $\left.\frac{1-\left(R_{2}+R_{x}\right) / \beta R_{p}}{\left(1+C_{p} / C_{2}\right)\left(1+R_{x} / R_{2}\right)}\right\}$ \\
\hline 7 & $f_{0}=\frac{\beta}{2 \pi} \sqrt{\frac{1}{C_{1} C_{2} R_{1} R_{2}}}$ & $f_{0}=\frac{\beta}{2 \pi}$ & $\frac{1}{C_{1} C_{2} R_{1} R_{2}}$ & $\left.\frac{1-\left(R_{2}+R_{x}\right) / R_{p}}{\left(1+C_{p} / C_{2}\right)\left(1+R_{x} / R_{2}\right)}\right\}$ \\
\hline 8 & $f_{0}=\frac{\beta}{2 \pi} \sqrt{\frac{1}{C_{1} C_{2} R_{2} R_{3}}}$ & $f_{0}=\frac{\beta}{2 \pi}$ & $\frac{1}{C_{1} C_{2} R_{2} R_{3}}$ & $\left.\frac{1}{\left(1+C_{p} / C_{2}\right)\left(1+R_{x} / R_{3}\right)}\right\}$ \\
\hline
\end{tabular}

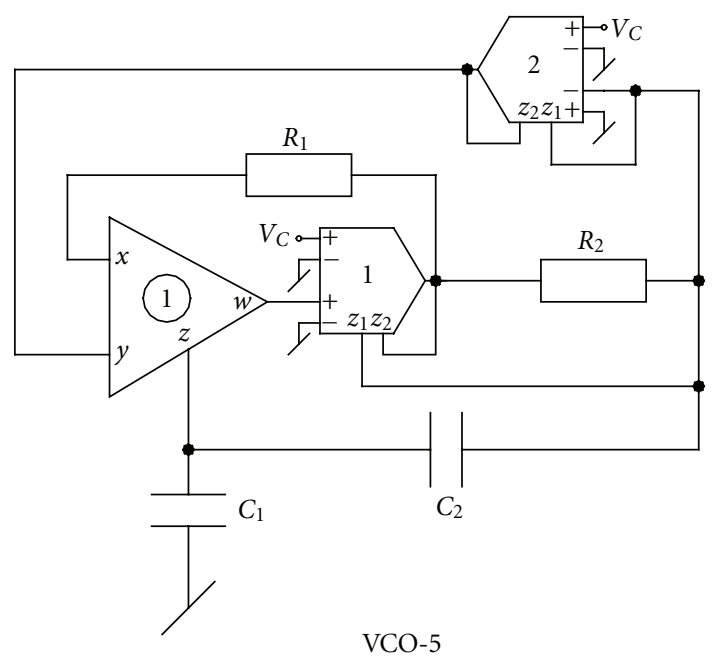

FIgURE 4: VCO derived from matrix $[A]_{3}\left(\mathrm{CO}: R_{1}=2 R_{2}\right.$, FO: $f_{0}=$ $\left.(\beta / 2 \pi) \sqrt{C_{1} C_{2} R_{1} R_{2}}\right)$.

also vary when the frequency is varied through the external control voltage $V_{C}$. This has indeed been the case of VCOs $1-5$. However, in case of VCOs 6, 7 and 8, the peak-to-peak output voltage has been found to be constant, $17 V_{p-p}$ in case of VCO 6 and 7 and $10 V_{p-p}$ in case of VCO- 8 when $V_{C}$ was varied from 1 to 10 volts. Thus, VCOs 6, 7, and 8 have been found to be superior to the other VCOs in this respect.

Some sample results of the proposed VCOs are shown in Figures $8(\mathrm{a})$ and $8(\mathrm{~b})$ which show the variation of oscillation frequency with control voltage $V_{C}$ for VCOs 1 and 8 , respectively.

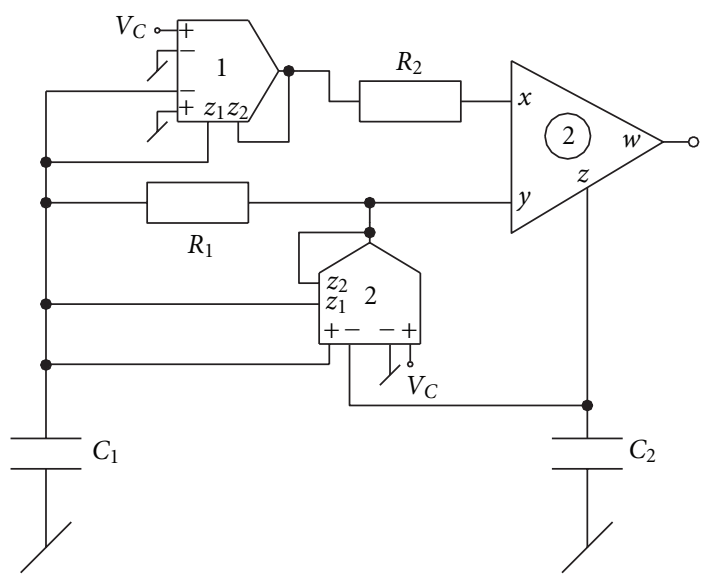

VCO-6

FIGURE 5: VCO derived from matrix $[A]_{4}\left(\mathrm{CO}: R_{1}=\left(C_{2} / C_{1}\right) R_{2}\right.$, FO: $\left.f_{0}=(\beta / 2 \pi) \sqrt{1 / C_{1} C_{2} R_{1} R_{2}}\right)$.

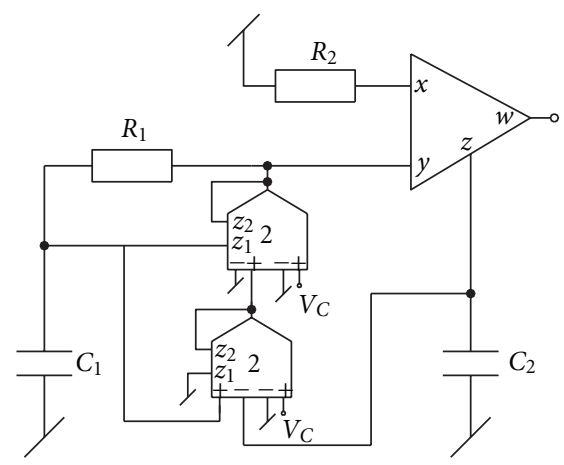

VCO-7

Figure 6: VCO derived from matrix $[A]_{5}\left(\mathrm{CO}: R_{1}=\left(C_{2} / C_{1}\right) R_{2}\right.$, FO: $\left.f_{0}=(\beta / 2 \pi) \sqrt{1 / C_{1} C_{2} R_{1} R_{2}}\right)$. 


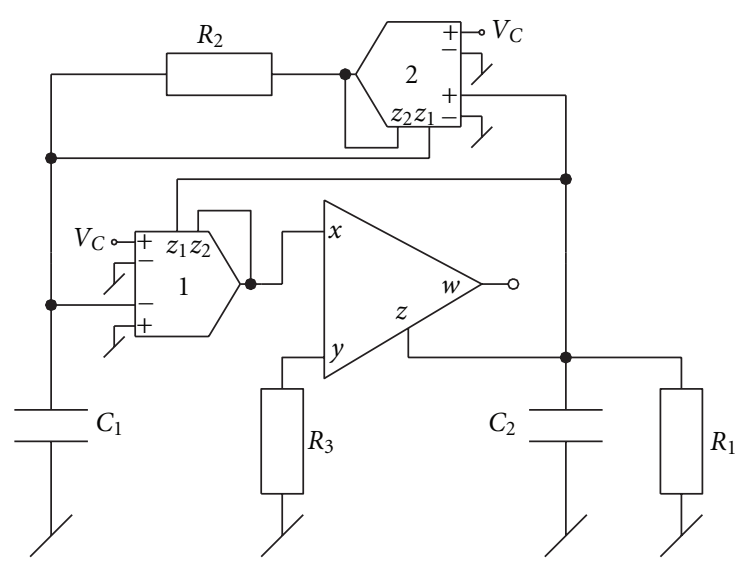

VCO-8

Figure 7: VCO derived from matrix $[A]_{6}\left(\mathrm{CO}: R_{1}=R_{3}\right.$, FO: $f_{0}=$ $\left.(\beta / 2 \pi) \sqrt{C_{1} C_{2} R_{2} R_{3}}\right)$.

\section{Concluding Remarks}

In this paper, we have presented a systematic derivation of eight new CFOA-AM-based VCOs, providing linear tuning laws. The workability of the new VCOs has been confirmed by the experimental results, based upon AD844 type CFOAs and AD534 type analog multipliers.

To the best knowledge of authors, the additional degree of freedom of summing a third input of the analog multiplier as prevalent in AD534 type multipliers has never been utilized earlier in the context of realization of the multiplierbased VCOs in particular and multiplier-based analog circuit design in general, and this is the major artifice which has led to the evolution of the new linear VCOs presented here, realizable with only a single CFOA.

When compared with the previously known linear CFOA-AM-based VCOs of $[11,18]$, the proposed circuits overcome the drawback of requiring a variable capacitance to adjust the condition of oscillation as prevalent in the VCOs of [18] and require the only one CFOA in contrast to the circuits of [11] requiring two CFOAs and not requiring use of several matched resistors as needed in the circuits of $[11,18]$.

Out of the eight circuits presented here, the VCO8 appears to be the best in terms of its performance (excellent linear tunability over two decades) coupled with the advantages of (i) noninteracting controls of CO and FO, (ii) an additional degree of freedom in scaling the frequency range by $R_{3}$ without disturbing condition of oscillation, (iii) employment of both grounded capacitors as advantageous for IC implementation see [27, 28] (and the references cited therein), and above all (iv) the novelty of retaining the feature of independent controllability of $\mathrm{CO}$ and FO even under the influence of various parasitics of the CFOA.

Lastly, it must be mentioned that in view of new CMOS CFOA and CMOS analog multiplier architectures continuously being evolved in the recent literature, the ideas of this paper could also be carried over to the design of completely CMOS-based linear VCOs; however, this requires

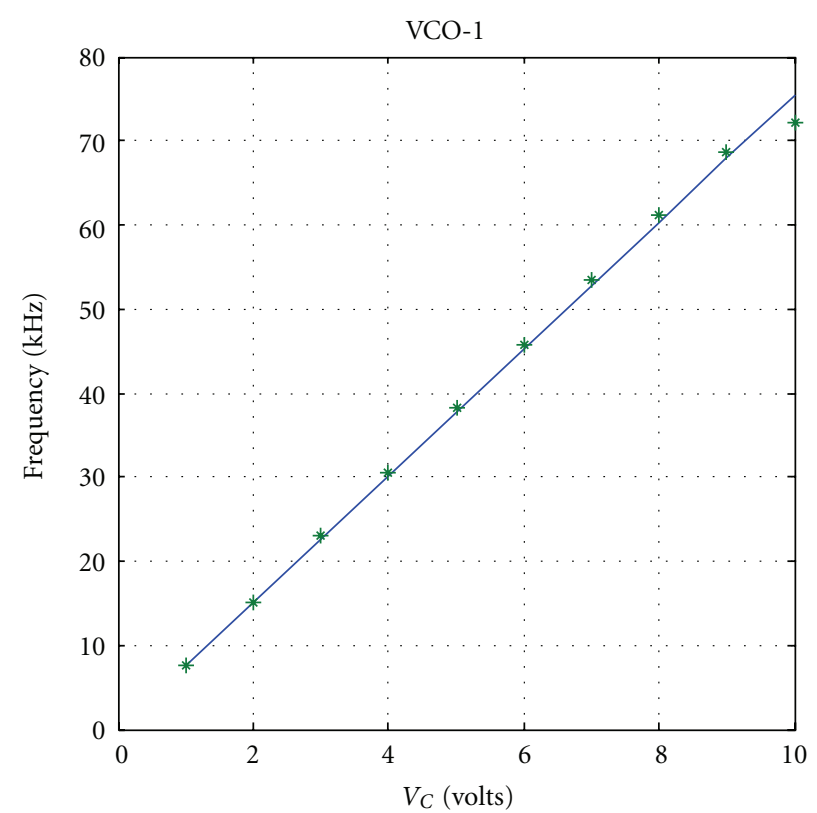

(a)

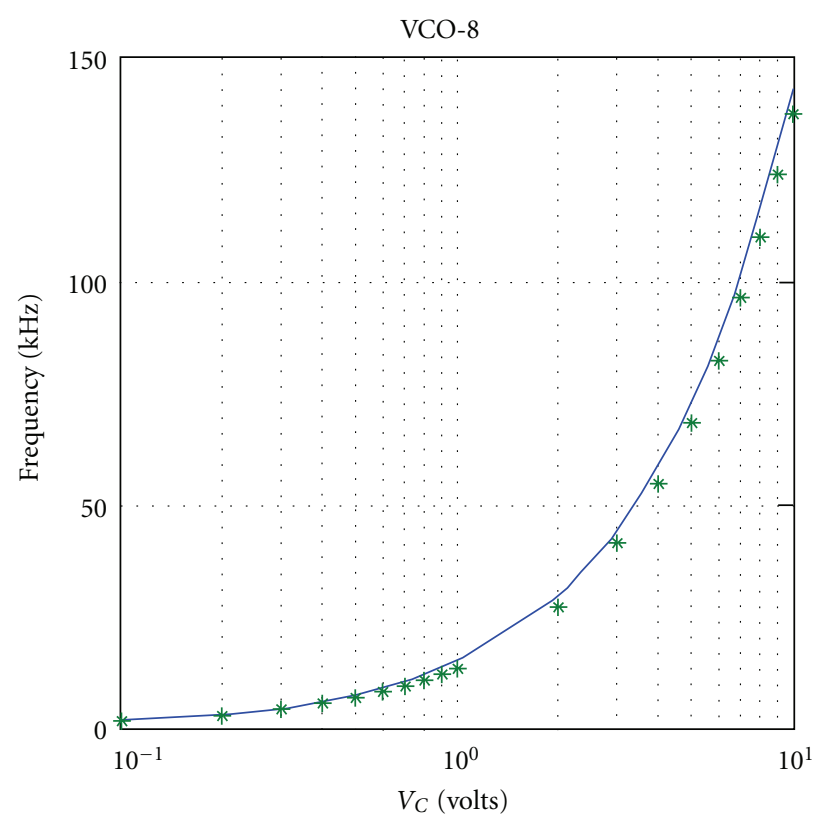

(b)

FIGURE 8: Experimental results of the VCOs: (a) Variation of frequency with $V_{C}$ for VCO-1, (b) Variation of frequency with $V_{C}$ for VCO-8.

considerable additional work and is, therefore, left for future investigations.

\section{References}

[1] J. W. Horng, "Current conveyors based allpass filters and quadrature oscillators employing grounded capacitors and resistors," Computers and Electrical Engineering, vol. 31, no. 1, pp. 81-92, 2005. 
[2] A. Toker, O. Çiçekoğlu, and H. Kuntman, "On the oscillator implementations using a single current feedback op-amp," Computers and Electrical Engineering, vol. 28, no. 5, pp. 375389, 2002.

[3] A. M. Soliman and A. S. Elwakil, "Wien oscillators using current conveyors," Computers and Electrical Engineering, vol. 25, no. 1, pp. 45-55, 1999.

[4] C. Toumazou and F. J. Lidgey, "Current feedback op-amps a blessing in disguise?" IEEE Circuits and Devices Magazine, vol. 10, no. 1, pp. 34-37, 1994.

[5] F. J. Lidgey and K. Hayatleh, "Current-feedback operational amplifiers and applications," Electronics and Communication Engineering Journal, vol. 9, no. 4, pp. 176-182, 1997.

[6] R. Senani, "Realization of a class of analog signal processing/signal generation circuits: novel configurations using current feedback op-amps," Frequenz, vol. 52, no. 9-10, pp. 196-206, 1998.

[7] S. S. Gupta, D. R. Bhaskar, and R. Senani, "New voltage controlled oscillators using CFOAs," AEU-International Journal of Electronics and Communications, vol. 63, no. 3, pp. 209-217, 2009.

[8] R. Senani and S. S. Gupta, "Synthesis of single-resistancecontrolled oscillators using CFOAs simple state-variable approach," IEE Proceedings Circuits, Devices and Systems, vol. 144, no. 2, pp. 104-106, 1997.

[9] S. S. Gupta and R. Senani, "State variable synthesis of singleresistance-controlled grounded capacitor oscillators using only two CFOAs additional new realizations," IEE Proceedings Circuits, Devices and Systems, vol. 145, no. 6, pp. 415-418, 1998.

[10] A. M. Soliman, "Current feedback operational amplifier based oscillators," Analog Integrated Circuits and Signal Processing, vol. 23, no. 1, pp. 45-55, 2000.

[11] D. R. Bhaskar, R. Senani, and A. K. Singh, "Linear sinusoidal VCOs: New configurations using current-feedback-op-amps," International Journal of Electronics, vol. 97, no. 3, pp. 263-272, 2010.

[12] R. Senani, D. R. Bhaskar, and M. P. Tripathi, "On the realization of linear sinusoidal VCOs," International Journal of Electronics, vol. 74, no. 5, pp. 727-733, 1993.

[13] R. Senani and D. R. Bhaskar, "New active-R sinusoidal VCOs with linear tuning laws," International Journal of Electronics, vol. 80, no. 1, pp. 57-61, 1996.

[14] D. R. Bhaskar and M. P. Tripathi, "Realization of novel linear sinusoidal VCOs," Analog Integrated Circuits and Signal Processing, vol. 24, no. 3, pp. 263-267, 2000.

[15] S. K. Saha, "Linear VCO with sine wave output," IEEE Transactions on Instrumentation and Measurement, vol. 35, no. 2, pp. 152-155, 1986.

[16] S. K. Saha and L. C. Jain, "Linear voltage controlled oscillator," IEEE Transactions on Instrumentation and Measurement, vol. 37, no. 1, pp. 148-150, 1988.

[17] V. P. Singh and S. K. Saha, "Linear sinusoidal VCO," International Journal of Electronics, vol. 65, no. 2, pp. 243-247, 1988.

[18] D. R. Bhaskar, R. Senani, A. K. Singh, and S. S. Gupta, "Two simple analog multiplier based linear VCOs using a single current feedback op-amp," Circuits and Systems, vol. 1, no. 1, pp. 1-4, 2010.

[19] V. K. Singh, R. K. Sharma, A. K. Singh, D. R. Bhaskar, and R. Senani, "Two new canonic single-CFOA oscillators with single resistor controls," IEEE Transactions on Circuits and Systems II, vol. 52, no. 12, pp. 860-864, 2005.
[20] R. Senani and V. K. Singh, "Synthesis of canonic single-resistance-controlled-oscillators using a single current-feedbackamplifier," IEE Proceedings Circuits, Devices and Systems, vol. 143, no. 1, pp. 71-72, 1996.

[21] S. I. Liu, C. S. Shih, and D. S. Wu, "Sinusoidal oscillators with single element control using a current-feedback amplifier," International Journal of Electronics, vol. 77, no. 6, pp. 10071013, 1994.

[22] P. A. Martínez, S. Celma, and J. Sabadell, "Designing sinusoidal oscillators with current-feedback amplifiers," International Journal of Electronics, vol. 80, no. 5, pp. 637-646, 1996.

[23] M. T. Abuelma'atti and S. M. Al-Shahrani, "A novel lowcomponent-count single-element-controlled sinusoidal oscillator using the CFOA pole," International Journal of Electronics, vol. 80, no. 6, pp. 747-752, 1996.

[24] M. T. Abuelma'atti and S. M. Al-Shahrani, "New CFOA-based sinusoidal oscillators," International Journal of Electronics, vol. 82, no. 1, pp. 27-32, 1997.

[25] M. T. Abuelma'atti and A. A. Farooqi, "A novel single-element controlled oscillator using the current-feedback-operational amplifier pole," Frequenz, vol. 50, no. 7-8, pp. 183-184, 1996.

[26] E. O. Güne and A. Toker, "On the realization of oscillators using state equations," AEU-Archiv fur Elektronik und Ubertragungstechnik, vol. 56, no. 5, pp. 317-326, 2002.

[27] M. Hribsek and R. W. Newcomb, "VCO controlled by one variable resistor," IEEE Transactions on Circuits and Systems, vol. 23, no. 3, pp. 166-169, 1976.

[28] S. S. Gupta and R. Senani, "Realisation of current-mode SRCOs using all grounded passive elements," Frequenz, vol. 57, no. 1-2, pp. 25-37, 2003. 

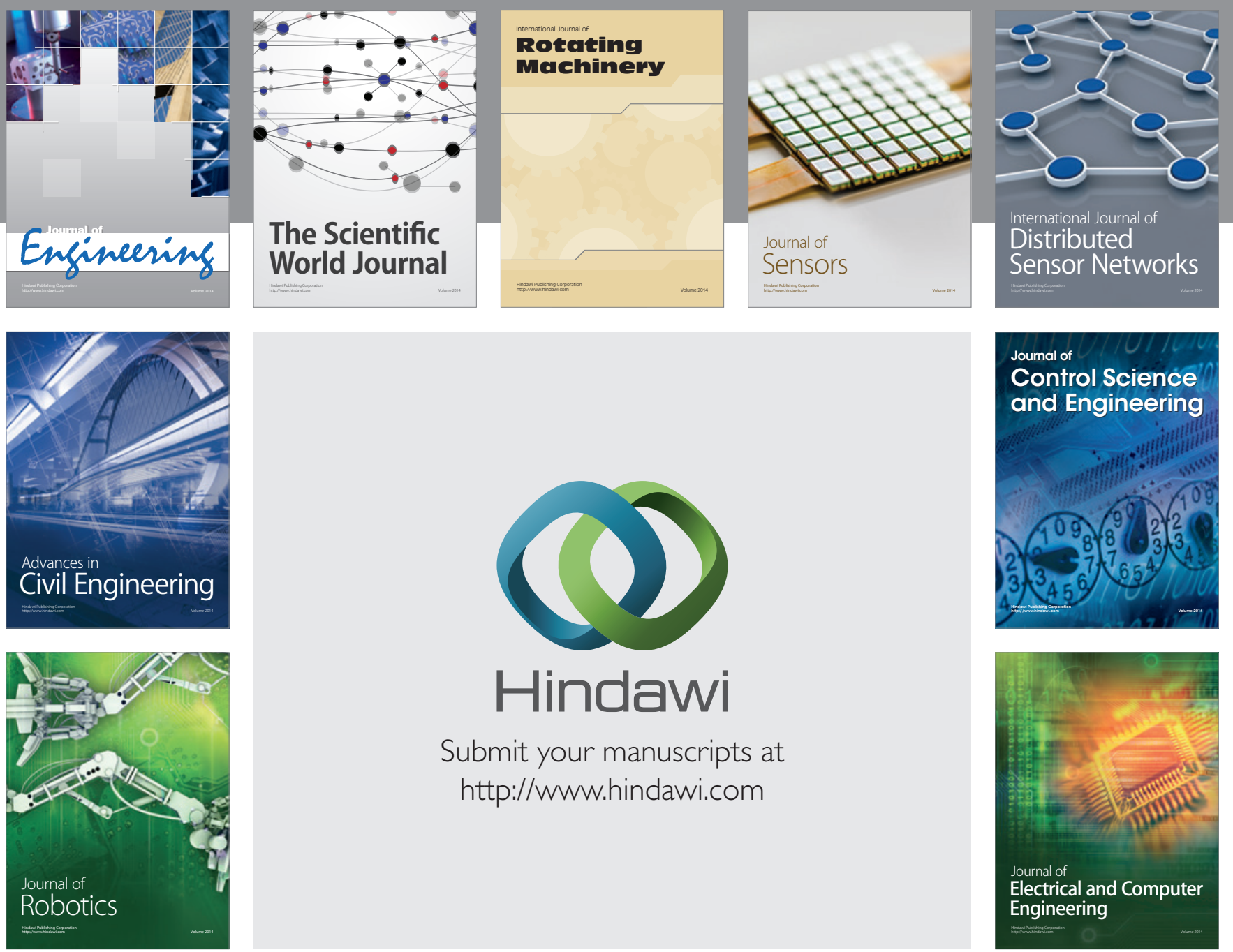

Submit your manuscripts at

http://www.hindawi.com
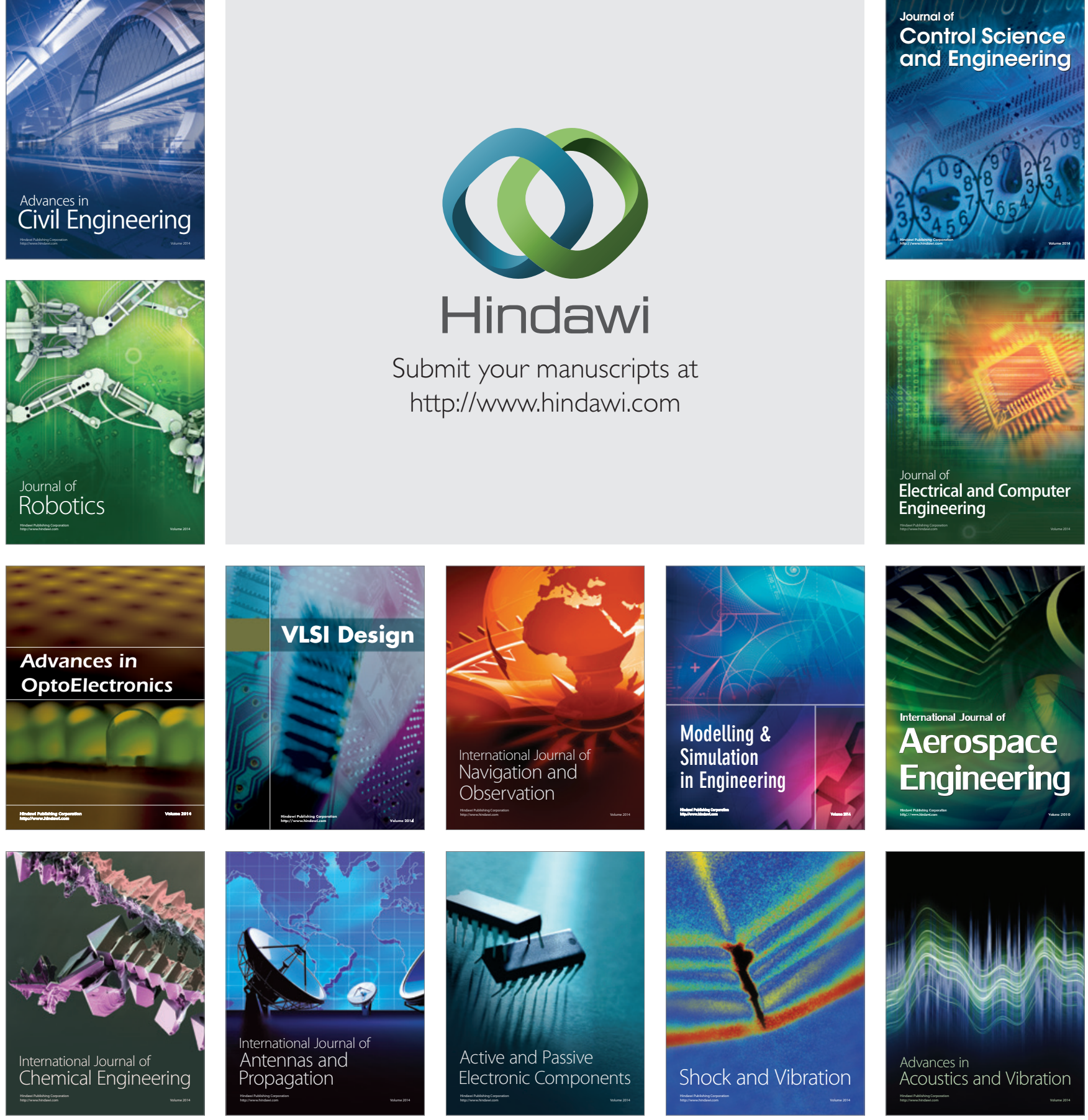